\title{
Fly Ash-Based Zeolite-Complexed Polyethylene-Glycol on an Interdigitated Electrode Surface for High-Performance Determination of Diabetes Mellitus
}

This article was published in the following Dove Press journal:

International Journal of Nanomedicine

Yan Chen

Ying Zhao

Yanjun Wang

Department of Endocrinology, The Second Hospital of Jilin University, Changchun, Jilin I3004I, People's Republic of China
Correspondence: Yanjun Wang Email jdeywyj@sina.com
Background: Diabetes is a complex metabolic disorder known to induce a high blood glucose level that fluctuates outside the normal range. Diabetes affects and damages the organs in the body and causes heart issues, blindness and kidney failure. Continuous monitoring is mandatory to keep the blood glucose level within a healthy range.

Materials and Methods: This research was focused on diagnosing diabetes mellitus on zeolite nanoparticle-polyethylene glycol complex-immobilized interdigitated electrode sensor (IDE) surfaces. Zeolite nanoparticles were extracted from the fly ash of a thermal power plant by alkaline extraction. The surface morphology of the synthesized nanoparticles was observed by field-emission scanning electron microscopy and transmission electron microscopy, and the presence of certain elements and the particle size were determined by energydispersive X-ray spectroscopy and particle size analysis, respectively.

Results: The crystalline PEG-zeolite nanoparticles were synthesized with a size of $40 \pm 10$ $\mathrm{nm}$ according to high-resolution microscopy. A particle size analyzer revealed the sizes of the fly ash and PEG-zeolite particles as $60 \pm 10 \mu \mathrm{m}$ and $50 \pm 10 \mathrm{~nm}$, respectively. The IDE surface was evaluated for its ability to display antifouling properties and sense glucose levels on the abovementioned nanoparticle-modified surface. Glucose oxidase was probed on the PEG-zeolite-modified IDE surface, and glucose was detected. PEG zeolite performed well with excellent antifouling properties on the IDE sensor surface and improved the glucose detection limit to $0.03 \mathrm{mg} / \mathrm{mL}$ from $0.08 \mathrm{mg} / \mathrm{mL}$, as determined by linear regressions $[\mathrm{y}=$ $5.365 \mathrm{x}-6.803 ; \mathrm{R}^{2}=0.9035$ (zeolite surface) and $\mathrm{y}=5.498 \mathrm{x}+5.914 \mathrm{R}^{2}=0.9061$ (PEGzeolite surface)]. This enhancement was $\sim 3$-fold, and sensitivities were found to be 0.03 and $0.06 \mathrm{mg} / \mathrm{mL}$ glucose for the PEG-zeolite- and zeolite-modified surfaces, respectively, showing a 2 -fold difference.

Conclusion: The excellent biocompatible surface modified by PEG zeolite exhibited high performance and is useful for medical diagnosis.

Keywords: blood glucose, dielectric sensor, biosensor, nanomaterial, nanoparticle

\section{Introduction}

The World Health Organization has predicted that the number of diabetes cases will rapidly increase in the upcoming years. Controlling the blood glucose level is currently challenging, and continuous monitoring is mandatory to keep diabetes in a controlled state. ${ }^{1}$ Improving the performance of blood glucose sensing systems by using a nanotechnological approach has helped improve the accuracy of diabetes 
diagnoses. ${ }^{2}$ The present investigation introduces a new strategy with a polyethylene glycol (PEG)-zeolite nanoparticle complex-modified interdigitated electrode (IDE) surface to quantify the level of glucose efficiently. IDE is one of the potential sensing systems that has been used widely and operates based on current-volt measurements. ${ }^{3-6}$ IDEs have di- or tri-electrodes that can be operated by AC or DC currents, making them suitable for industrial and point-of-care applications. ${ }^{7-11}$ Furthermore, the surface of IDEs can be modified physically or chemically, and under physical modification, nanoparticles have been widely applied and demonstrated high-performance detection capabilities. ${ }^{8,12-17}$ These surface modifications play a crucial role in the dipole moment that occurs with molecular immobilization. Upon high biomolecular attachment due to the enhanced surface area created by the nanoparticle, an increased amount of interactions between the probe and target can occur. Therefore, pathways for the direct current flow are generated, and the resistance is steadily enhanced. The formation of a molecular complex induces vibrations and causes displacement of the charge between the dielectrode gaps. The created charge displacement with a potential confinement is called a "dipole moment".

A handful of metallic and nonmetallic nanoparticles have been reported, and among these, zeolite particles have a unique composition. ${ }^{8,18,19}$ Zeolite nanoparticles exhibit hydrophobicity and are porous with a crystalline nature of aluminosilicates and a high pore volume and surface area. Due to the nontoxic effect of zeolite nanoparticles on human cells, zeolite nanoparticles have gained increasing attention in the area of medicinal applications, such as bioimaging and drug delivery. ${ }^{20}$ In biosensing applications, the signal-to-noise ratio is high due to the ease pf protein adsorption onto the surface of zeolites. Rahimi et $\mathrm{al}^{21}$ analyzed the affinity of plasma proteins to zeolite nanoparticles and found high adsorption of apolipoprotein and fibrinogen on the surface of the zeolite nanoparticles. Moreover, when zeolite nanoparticles were injected into the blood serum, the proteins quickly bound to the surface of the particle. To overcome this issue, this research synthesized polyethylene glycol (PEG)-modified zeolite (PEG-zeolite) to make the nanoparticles more biocompatible with antibiofouling properties.

PEG is a chemically inert, inexpensive, water soluble, coiled polymer with repeating units of ethylene and is considered to be a versatile material as approved by the Food and Drug Administration for several applications. ${ }^{22,23}$ PEGylated nanoparticles have proven to have resistance to biofouling proteins due to their flexible and hydrophilic nature. Hossain et $\mathrm{al}^{24}$ observed limited binding of bovine serum albumin on PEG-modified silica surfaces. More specifically, the combination of short and long polymers showed a greater impact on preventing nonspecific protein adsorption on the sensor surfaces than when using a polymer of one length. ${ }^{25}$ Additionally, PEG-modified surfaces were found to improve specific biomolecular interactions. ${ }^{26}$ Lakshmipriya et $\mathrm{al}^{27}$ used a black copolymer, poly(ethylene glycol)-b-poly(acrylic acid), on (3-aminopropyl)triethoxysilane-modified silica surfaces to enhance the detection of blood clotting factor IX protein. In addition, the construction of PEG and a ligand on gold surfaces improved the detection of thrombin protein. ${ }^{28}$ The hydrophilic outer area of PEG has potential for application in receptor-mediated gene and drug delivery systems, bioassays and imaging processes. ${ }^{29}$ Considering all these positive points of PEGylated particles, this study synthesized PEGylated zeolite nanoparticles (PEG-zeolite) from waste coal fly ash from a thermal power plant (India), which was desirable due to the enriched alumina and silica contents of this particular coal fly ash. Several studies are ongoing with the utilization of flashes from various sources for downstream applications. ${ }^{30}$ Since silica and alumina are the major compounds in coal fly ash, the synthesized zeolite and PEG-zeolite nanoparticles were modified on the IDE sensor surface and utilized for glucose sensing.

\section{Materials and Methods}

\section{Reagents and Biomolecules}

The coal fly ash was collected from the Thermal Power Plant, Tamil Nadu, India. Sulfuric acid and sodium hydroxide were purchased from Sigma Aldrich (USA). Whatman filter paper was purchased from Thermo Fisher Scientific (USA). 3-(Aminopropyl)-tryethoxysilane (APTES), bovine serum albumin (BSA), glucose oxidase (GOx), glucose, glutaraldehyde, polyethylene glycol and phosphate buffered saline (PBS, pH 7.4) were obtained from Sigma Aldrich (USA).

\section{Synthesis of Zeolite and PEG-Zeolite Nanoparticles}

Sodium aluminosilicate was extracted from the coal fly ash to synthesize the zeolite nanoparticles. Before extracting sodium aluminosilicate, the fly ash was first acid leached 
to remove minerals and other contaminants. For acid leaching, $500 \mathrm{~mL}$ of $10 \% \mathrm{H}_{2} \mathrm{SO}_{4}$ was mixed with 50 $\mathrm{g}$ of fly ash and heated at $100^{\circ} \mathrm{C}$ with constant stirring. Then, the residue was separated by filtration and washed several times with water until it reached $\mathrm{pH} 7$. The acidleached fly ash was used to extract sodium aluminosilicate. The acid-leached ash was dissolved in $1 \mathrm{~L}$ of 2.5 $\mathrm{M}$ sodium hydroxide and heated at $100^{\circ} \mathrm{C}$ for $4 \mathrm{~h}$ to properly dissolve the silica and alumina. Then, the mixture was filtered through Whatman filter paper, and the solution of sodium aluminosilicate was used to synthesize the zeolite nanoparticles.

To synthesize the zeolite nanoparticles, $1 \mathrm{~L}$ of extracted sodium aluminosilicate was titrated by $2 \mathrm{M} \mathrm{H}_{2}$ $\mathrm{SO}_{4}$ at $60^{\circ} \mathrm{C}$ under constant stirring until it reached $\mathrm{pH} 7$. At $\mathrm{pH} 7$, formation of a clear gel was observed, and the gel was stirred continuously for $18 \mathrm{~h}$ to obtain uniform zeolite particles. Then, the gel was separated, washed with water several times, and dried at $60^{\circ} \mathrm{C}$ to obtain zeolite nanoparticles. Finally, the dried particles were calcinated at $400^{\circ} \mathrm{C}$ for $2 \mathrm{~h}$ to obtain the desired zeolite nanoparticles. To synthesize PEG-zeolite nanoparticles, $0.5 \%$ PEG was suspended in the extracted sodium aluminosilicate and then titrated by $\mathrm{H}_{2} \mathrm{SO}_{4}$. The subsequent procedures were the same as those mentioned above to obtain the PEG zeolite.

\section{Characterization of Synthesized PEG-Zeolite Nanoparticles}

The morphological and compositional elements of the synthesized particles were studied by field emission scanning electron microscopy (FESEM, Hitachi, S-4300 SE, Japan) and transmission electron microscopy (TEM, Transmission JEOL JEM 2100F). The FESEM/EDX spectrum was used to find the compositional elements of the synthesized PEG zeolite. Further confirmation experiments were conducted by a particle size analyzer.

\section{Interdigitated Electrode Fabrication Process}

Interdigitated electrode (IDE) fabrication was performed by utilizing the traditional wet-etching method. ${ }^{13}$ The IDE base was a silica wafer was cleaned by utilizing the solutions of RCA1 and RCA2 to reduce unnecessary deposits on the wafer surface. Furthermore, the wafer was processed at $500^{\circ} \mathrm{C}$ (thermal oxidation) for $1 \mathrm{~h}$ to oxidize the wafer layer, and a silicon dioxide layer was formed. On the oxidized layer, an aluminum coil was used to deposit aluminum using a thermal evaporator. The conventional photolithography method was used to create the electrode pattern on the aluminum substrate. Furthermore, the substrate was exposed to UV light to transfer the IDE pattern onto a photoresistor. The unexposed area of the IDE was eliminated by dipping the substrate in the photoresistor developer, and the moisture content was removed by baking the substrate at $110^{\circ} \mathrm{C}(1$ min). In the final step, the unexposed layer of aluminum was etched by dipping the IDE in an etchant solution. The prepared IDE was cleaned with acetone followed by distilled water and kept in a desiccator. The IDE surface profile was monitored under a 3D nanoprofiler and with high-power microscopy for confirmation.

\section{Blocking Effect of PEG Zeolite on the Amine-GLU-Modified IDE Surface}

To evaluate the blocking effect of PEG zeolite on the amine-GLU-modified IDE surface, the sensor surface was initially modified with the amine by using APTES. Before this modification, the sensor surface was treated with an alkaline solution $(\mathrm{KOH})$ for $1 \mathrm{~min}$. Then, $2 \%$ APTES diluted in ethanol was added to the sensor surface and kept for several hours to modify the surface with amine groups. Then, 2.5\% glutaraldehyde (GLU) diluted in water was added to the amine-modified surface by incubating for $1 \mathrm{~h}$. These modified surfaces were placed on a two-pointed probe station for the current measurement. Then, PEG-zeolite nanoparticles $(1 \mathrm{mg} / \mathrm{mL})$ were added to the surface, and the changes in the current were recorded. For comparison, BSA (1 mg/mL), commercial $\mathrm{SiO}_{2}(1 \mathrm{mg} / \mathrm{mL})$ and synthesized zeolite were used in different experiments on APTES-GLU-modified surfaces.

\section{Specific Detection of Glucose on PEG-Zeolite Modified Surfaces}

After analyzing the attachment of nanoparticles on the IDE sensor, glucose was quantified on the IDE surface with PEGzeolite and compared with the zeolite-modified surface. The following processes were performed to determine glucose on the PEG-zeolite-modified IDE surface: (i) the IDE surface was modified with amine by $2 \%$ APTES diluted in ethanol; (ii) $2 \%$ GLU was added to the APTES-modified surface; (iii) $1 \mu \mathrm{M}$ GOx diluted in PBS buffer was allowed to bind to the GLU surface; (iv) the remaining surface was blocked by $1 \mathrm{mg} / \mathrm{mL}$ PEG zeolite, which covered the remaining amine and GLU sites; and (v) finally, glucose was determined on 
$\mathrm{G}$ the Ox-modified surfaces. For comparison, a similar experiment was carried out with zeolite instead of PEG zeolite.

\section{Glucose Detection Limit and Specificity on PEG-Zeolite-Modified IDE Surface: High-Performance Analysis}

To check the glucose detection limit, first, the IDE surface was modified with amines, GLU, and then GOx. Then, the remaining sensor surface was blocked by PEG-zeolite or zeolite nanoparticles as described above. Different concentrations of glucose ( 0.03 to $0.5 \mathrm{mg} / \mathrm{mL}$ ) were then passed on the surface independently, and the changes in current were recorded to observe the differences after glucose immobilization. Similar experiments were conducted at $0.5 \mathrm{mg} / \mathrm{mL}$ using fructose and galactose instead of glucose at the desired concentration to determine the detection specificity.

\section{Results and Discussion}

This study was performed to analyze glucose levels to diagnose diabetes mellitus. An interdigitated electrode (IDE) sensor was used for this diagnosis by modifying the surface with the synthesized nanoparticles along with chemical functionalization (Figure 1). Upon immobilizing the molecules, the operating current-volt of the system change due to changes in the dipole moment (Figure 1; inset). Coal fly ash is an abundant residue from thermal power plants and is utilized to extract zeolite nanoparticles. Zeolite nanoparticles can be used for application as catalysts and in gas separation and adsorption processes. ${ }^{31}$ In this work, zeolite nanoparticles were used for surface modification in biosensing applications to determine and quantify the level of glucose for diagnosing diabetes. The zeolite particles were isolated by the alkaline extraction method from coal fly ash using a multistep process. The sodium silicate solution obtained from this process was used as a silica-aluminum precursor to synthesize zeolite via a sol-gel method using polyethylene glycol (PEG) as the surfactant and sulfuric acid as the catalyst. During this process, the participation of PEG in the hydrolysis reaction and condensation along with sodium aluminosilicate led to the production of PEGgrafted zeolite nanoparticles. Before starting the main set of experiments, the produced nanoparticles were characterized.

\section{Characterization of PEG-Zeolite Nanoparticles}

For the purpose of enhanced site blocking with high antibiofouling properties, PEG was complexed with zeolite nanoparticles produced from coal fly ash by extracting sodium aluminosilicate. The morphological and elemental characteristics of the raw fly ash, synthesized zeolite and PEG zeolite were studied by different analyses.
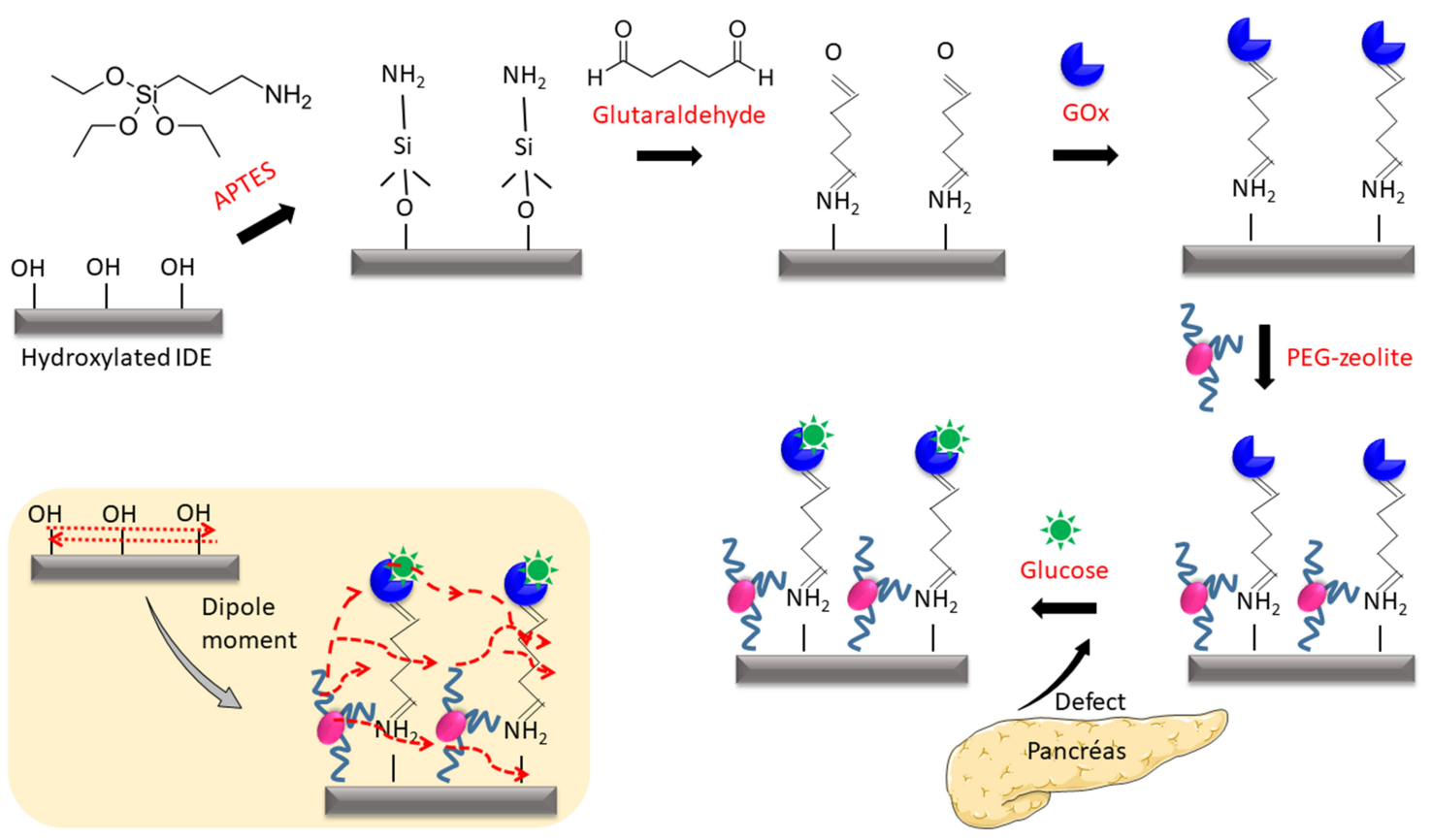

Figure I Schematic representation of glucose detection on a PEG-zeolite-modified IDE sensor. PEG zeolite was deposited on APTES-GLU-modified IDE surfaces after GOx was bonded with GLU. This surface was utilized to detect glucose. PEG zeolite was used as the blocking agent and provided proper orientation to GOx for improving glucose detection. The figure inset displays the dipole moment on the surface. 


\section{Field-Emission Scanning Electron Microscopy (FESEM) and Energy-Dispersive X-Ray Spectroscopy (EDX) Analyses}

Figure 2A shows the FESEM image of the raw fly ash, and from this figure, it was noticed that the raw fly ash particles had a spherical shape with a smooth surface, and the diameter was found to be in the range of $40 \pm 20$ $\mu \mathrm{m}$. The sizes of the particles were estimated by comparing the scale bar generated during the measurements. Moreover, some agglomeration of small particles was noticed within the fly ash. Figure 2B displays the synthesized PEG-zeolite nanoparticles and shows uniform spherical shaped particles with an average size in the range of $40 \pm 10 \mathrm{~nm}$. Figure $2 \mathrm{C}$ is the image and EDX spectra for the PEG zeolite. The EDX spectra reveal the presence of silica and alumina in the PEG- zeolite nanoparticles. The presence of sodium (Na) might originate from the purification process of alkali extraction.

\section{Transmission Electron Microscopy (TEM)}

The PEG-zeolite nanoparticles were also analyzed by TEM to observe the surface morphology. Figure 2D represents the TEM image of the PEG-zeolite nanoparticle, and it was noticed that uniformly sized PEG-zeolite nanoparticles at the nanometer level were generated and that the pores of the PEG-zeolite nanoparticles were loosely arranged in a regular array, all connected with each other with a uniform orientation. The predicted size from TEM matched the size measured by FESEM analysis. The characterized particles were attached on the IDE surface to minimize biofouling and enhance the performance of the sensor.
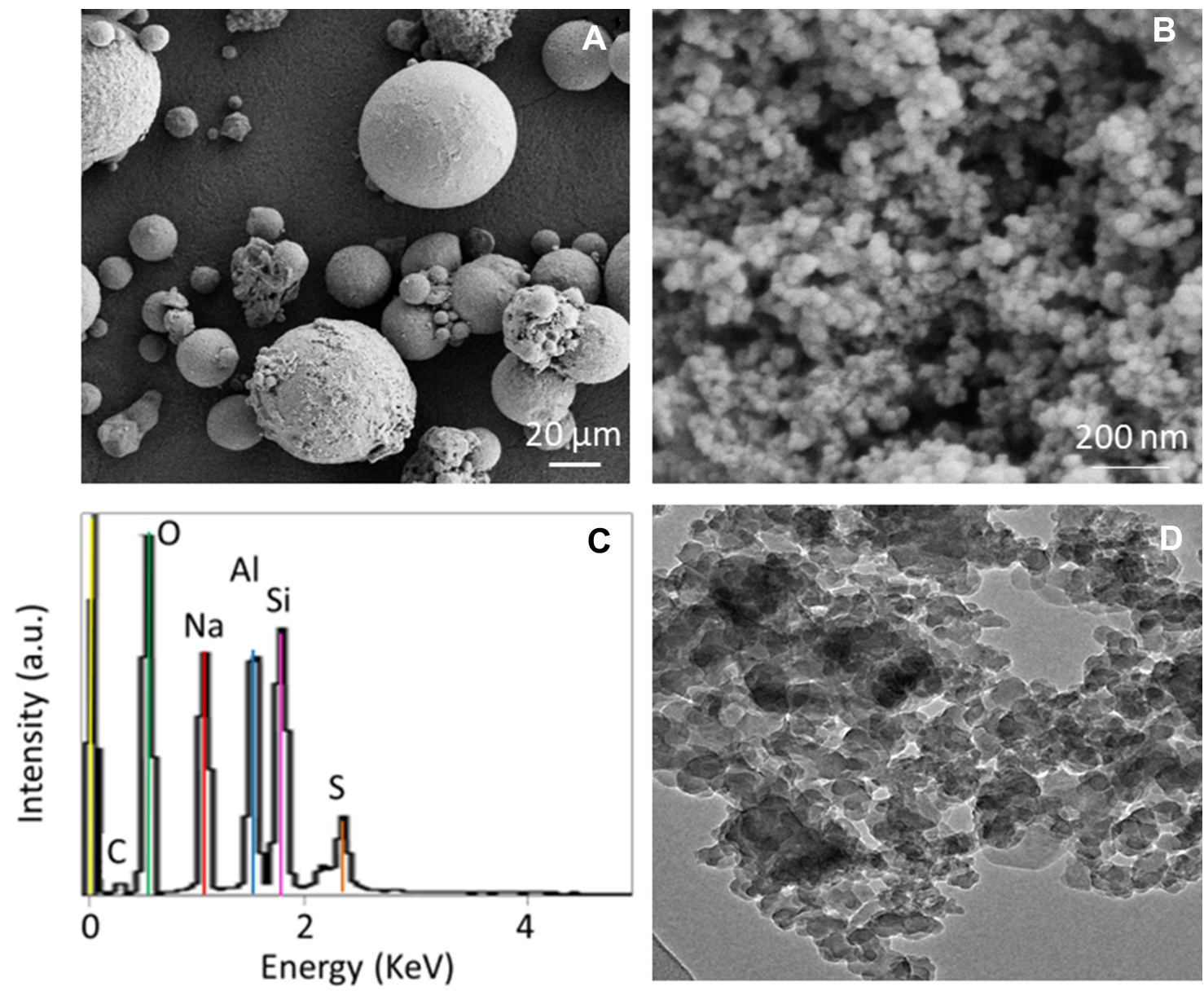

Figure 2 (A) FESEM image of raw fly ash particles. Particles show spherical shapes with different diameters within the range of $60 \pm 10 \mu m$. (B) FESEM image of the extracted PEG-zeolite nanoparticles. Particles show a spherical shape with a uniform distribution, and the size of the particles is in the range of $50 \pm 10 \mathrm{~nm}$. (C) EDX analysis of PEGzeolite nanoparticles. The presence of silica and alumina indicates the formation of zeolite nanoparticles. (D) TEM image of PEG-zeolite nanoparticles. Nanoparticles are clearly distributed with uniform size. 


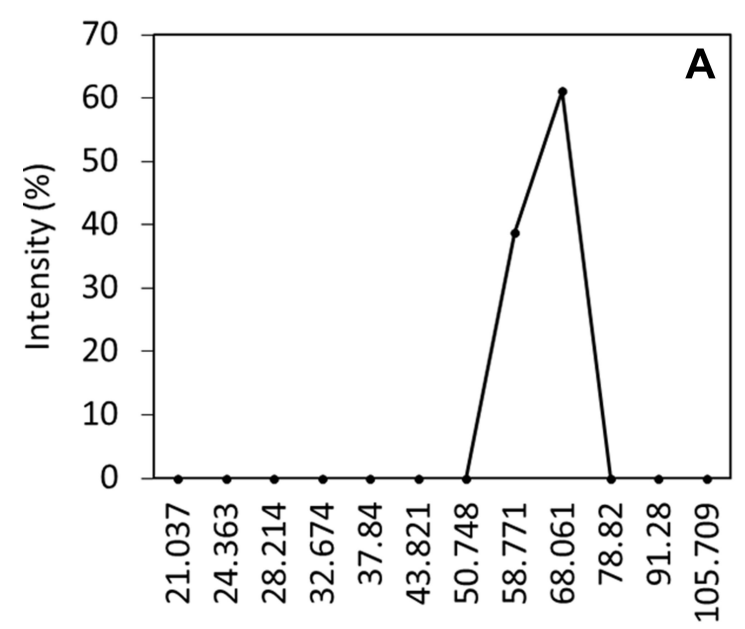

Size $(\mu \mathrm{m})$

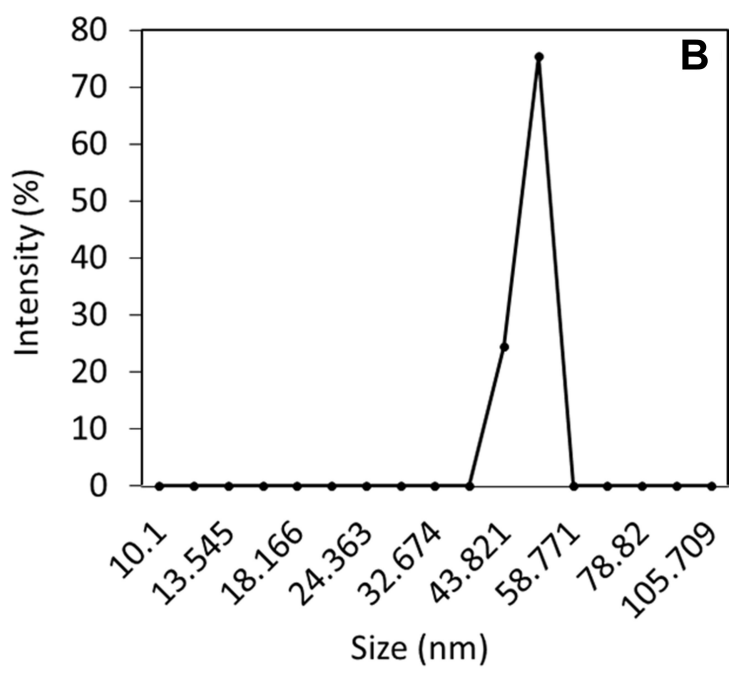

Figure 3 Particle size analysis. (A) Fly ash; (B) PEG-zeolite. Both fly ash and PEG-zeolite particles were yielding the average sizes of $60 \pm 10 \mu \mathrm{m}$ and $50 \pm I 0 \mathrm{~nm}$, respectively. A drastic size reduction of the particles from the micro- to nanoscale was noticed.

\section{Particle Size Analysis}

A particle size analyzer was also used to test the size of fly ash and the prepared PEG zeolite, which presented average sizes of $60 \pm 10 \mu \mathrm{m}$ and $50 \pm 10 \mathrm{~nm}$, respectively. Based on these results, it seems that agglomeration did not truly occur (Figure 3A and B). Furthermore, there was a drastic size reduction in particles from the micro- to nanoscale after the alkaline extraction performed in this study.

\section{Fabrication and Surface Analysis of the IDE}

Fabrication of the IDE surface was performed by the wetetching method as described in the Methods section, and the important process steps are displayed in Figure 4A. The surface dimensions of the IDE surface were designed by AutoCAD and have a length of $7500 \mu \mathrm{m}$, a width of 4100 $\mu \mathrm{m}, 20$ finger-gap pairs, a gap size of $10 \mu \mathrm{m}$, an electrode
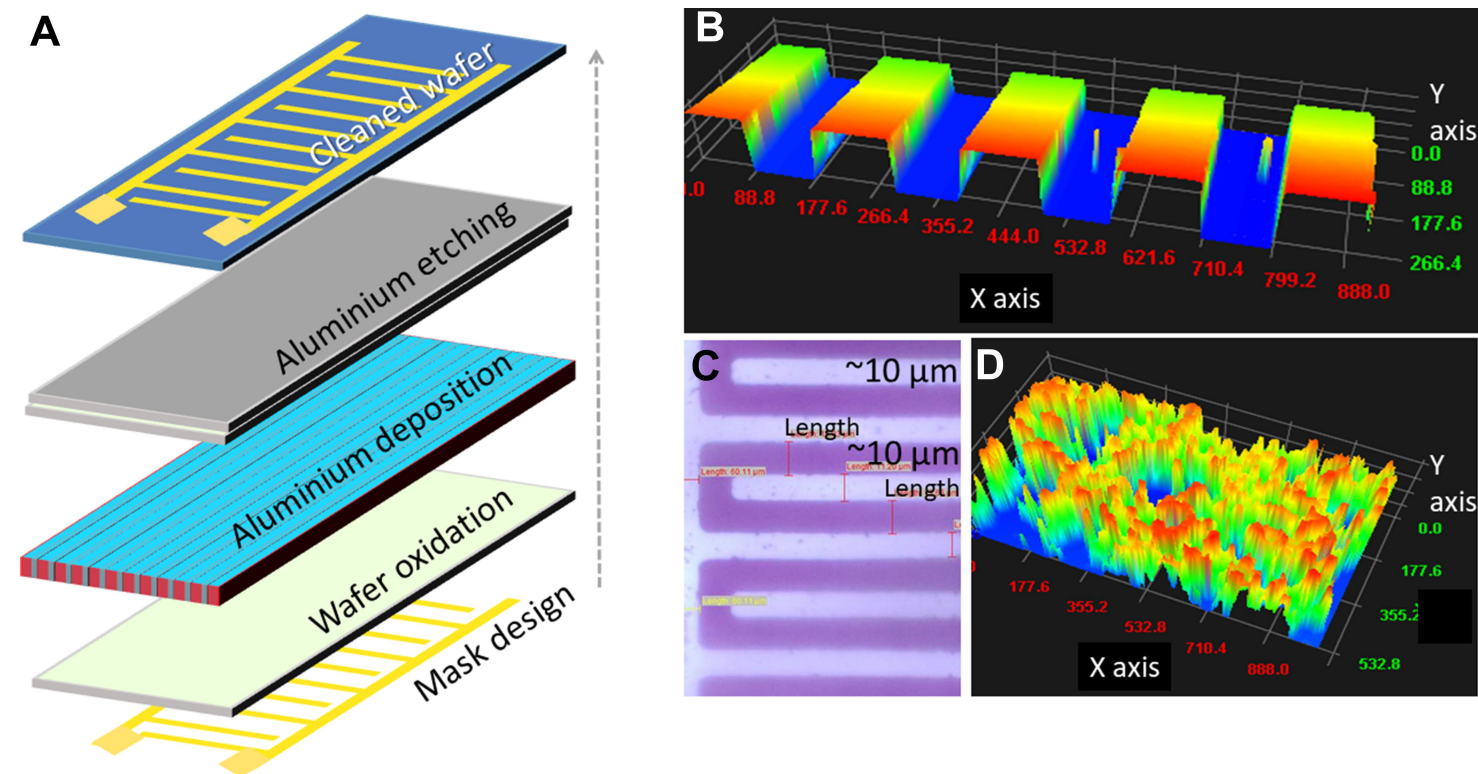

Figure 4 (A) Mask design and fabrication process. Important procedures are displayed. (B) 3D nanoprofiler image on the IDE surface. (C) High-power microscopy image of the IDE surface. (D) 3D nanoprofiler image of the IDE surface immobilized with PEG-zeolite nanoparticles. 
size of $10 \mu \mathrm{m}$, an electrode thickness of $40 \mathrm{~nm}$, and a finger length of $4000 \mu \mathrm{m}$. The properly arranged finger and gap regions were observed under the 3D nanoprofiler, confirming the uniformity (Figure 4B). Supporting confirmation was carried out by high-power microscopy, which attested to the presence of finger and gap regions $10 \mu \mathrm{m}$ in size (Figure 4C). With these confirmations, further experiments were conducted by attaching nanoparticles on the IDE surface.

\section{Antibiofouling Effect of PEG Zeolite on Amine-GLU Modified IDE Surfaces}

An improved antibiofouling sensor is mandatory to improve biomolecular identification. The use of a suitable blocking agent has been found to reduce the signal-to-noise ratio and improve the limit of detection of sensors. ${ }^{32,33}$ In the current investigation, PEG zeolite was used as the antifouling agent on the amine-GLU-modified IDE sensor surface to detect the level of glucose to diagnose diabetes mellitus. To evaluate the antifouling effect of PEG zeolite, the binding affinity of the synthesized zeolite on our chemically modified surfaces was tested. For that, PEG zeolite was immobilized on amine-
GLU-modified IDE surfaces, and the obtained results were compared with those of zeolite (synthesized from fly ash), commercial $\mathrm{SiO}_{2}$, and the common blocking agent BSA immobilized on the surface. As shown in Figure 5A-D, PEG-zeolite binding was strong on the amine-GLUmodified surface and showed the highest difference in current compared with those of the zeolite-, BSA-, and commercial $\mathrm{SiO}_{2}$-modified surfaces. In the case of BSA, the current difference was 3.97 times that of the base surface (Figure $5 \mathrm{~A}$ ), whereas that of commercial $\mathrm{SiO}_{2}$ was 1.84 times (Figure 5B), that of zeolite was 8.1 times (Figure 5C) and that of PEG zeolite was 20 times that of the base surface (Figure 5D). Compared with that of commercial $\mathrm{SiO}_{2}$, PEGzeolite binding was found to be 10 times higher, and zeolite binding was 2.5 times lower than that of PEG zeolite. These differences in binding were due to the strong binding affinity of PEG zeolite on the amine-GLU surfaces. The strong attachment of PEG zeolite was confirmed by observation under a 3D nanoprofile imager (Figure 4D). Previously, it was proven that the polymer [poly(ethylene glycol)-b-poly (acrylic acid)] PEG-b-PAAc binds on amine-GLU-modified
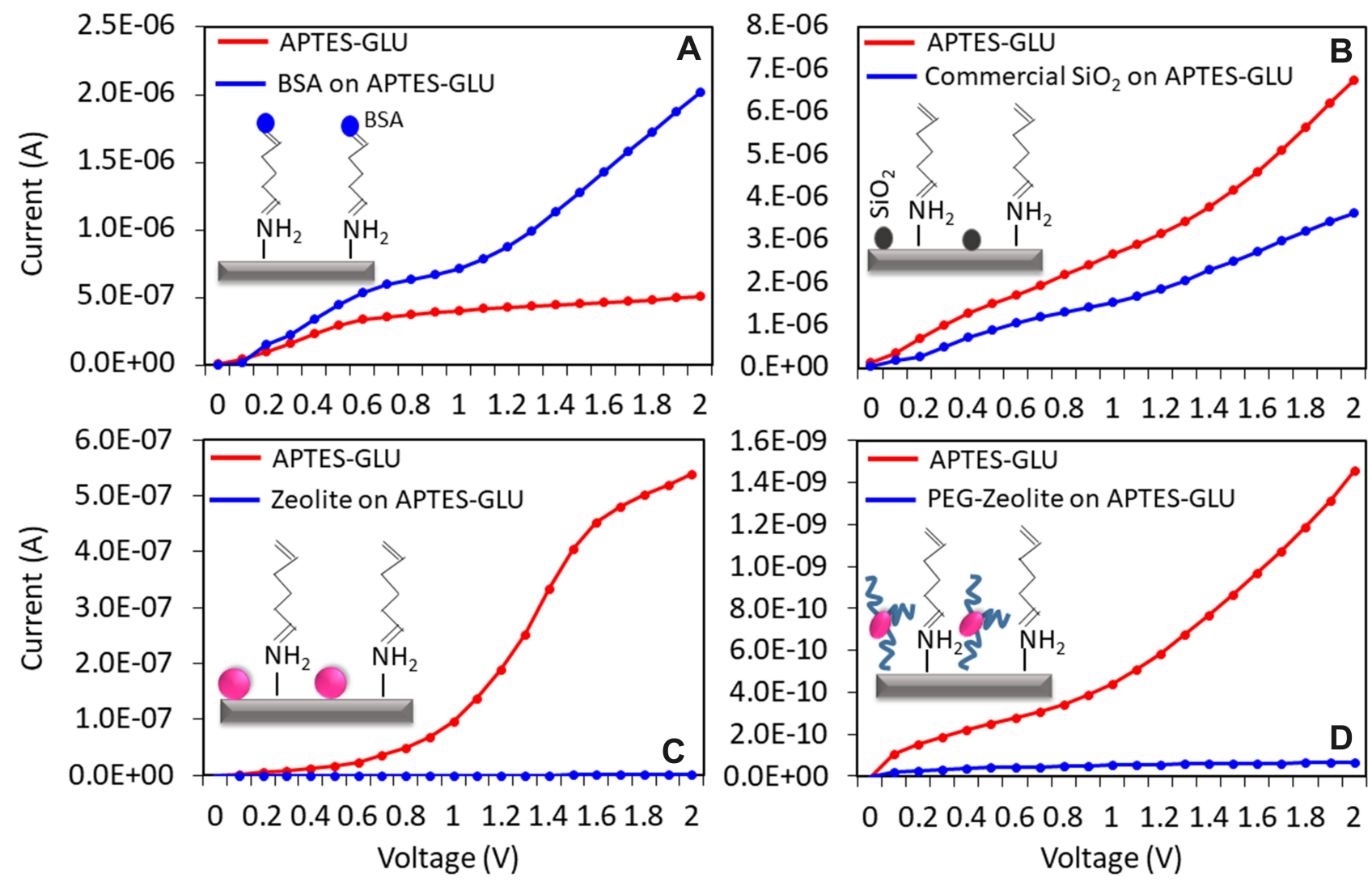

Figure 5 Comparison of blocking efficiency on APTES-GLU-modified IDE surfaces. On APTES-GLU-modified surfaces, different molecules/particles were placed, and the blocking efficiency was checked. (A) BSA; (B) Commercial $\mathrm{SiO}_{2}$; (C) Zeolite; (D) PEG zeolite. PEG zeolite shows a higher current response than that of the other surface modifiers, indicating its strong binding affinity on APTES-GLU-modified surfaces. Figure insets display the diagrammatic representations. 
silica surfaces, reduces nonspecific protein and gold nanoparticle binding and enhances the detection limit of human clotting factor IX. ${ }^{27}$

\section{Determination of Glucose on PEG-Zeolite- and Zeolite-Immobilized IDE Surfaces}

Since zeolite and PEG zeolite showed a strong binding affinity on the chemically modified IDE sensor surfaces, this study initially detected $0.25 \mathrm{mg} / \mathrm{mL}$ glucose on PEGzeolite and zeolite-modified IDE surfaces. For this study, GOx was immobilized on the APTES-GLU-modified surface (APTES-GLU-GOx), and then zeolite or PEG zeolite was allowed to bind (block) the remaining sites on the sensing surfaces. Then, glucose was allowed to interact with GOx. Before passing the glucose over the GOx, just 1 nM BSA was passed over the sensing surface to check the remaining available sites on the sensing surface. By comparing Figure 6A and B, PEG-zeolite binding was higher than zeolite binding, and BSA blocking showed a significant change in current with the zeolite-modified surface, whereas into change could not be observed on the PEG-zeolitemodified surface. This difference was due to the binding of BSA on the free GLU sites, few of which were present in the case of PEG zeolite. Moreover, PEG may have covered the free GLU spaces so that BSA could not bind to any open sites. This result confirmed the effective antibiofouling of PEG-zeolite compared with that of zeolite alone. In addition, $0.25 \mathrm{mg} / \mathrm{mL}$ glucose showed a higher current increment on the PEG-zeolite-modified surface than on the zeolitemodified surface. Moreover, in the process of glucose immobilization, a 5.6 times current (from $8.44 \mathrm{E}^{-5}$ to $4.77 \mathrm{E}^{-4} \mathrm{~A}$ ) increment was noticed in the case of PEG-zeolite modification; on the other hand, in the case of zeolite modification, the current increased to 5.1 times (from $2.55 \mathrm{E}^{-5}$ to $1.31 \mathrm{E}^{-4}$ A) that prior to glucose immobilization. Even though the increasing currents in both cases were close, the surface with zeolite may have given a false positive response due to the binding of glucose on GLU surfaces, and additional blocking agents, such as BSA, may need to be used to cover the remaining GLU surfaces. In the case of the PEG-zeolitemodified surface, the clear binding of glucose on GOx with the confident increment in current without any nonspecificity was apparent. This might have been due to the proper orientation of PEG zeolite on the amine-modified surface allowing for the proper and stable interaction of glucose on the GOx surface.

\section{Limit of Glucose Detection on PEG-Zeolite-Modified Surfaces}

After finding the optimal antifouling effect of zeolite and PEG zeolite, to check the limit of detection, different concentrations of glucose $(0.03$ to $0.5 \mathrm{mg} / \mathrm{mL})$ interacted on GOx-modified surfaces in the presence of zeolite or PEG zeolite. As shown in Figure 7, in both cases, with increasing glucose concentration, the current also increased. In the case of zeolite, the glucose levels of 0.03 and $0.06 \mathrm{mg} / \mathrm{mL}$ did not show a prominent increment
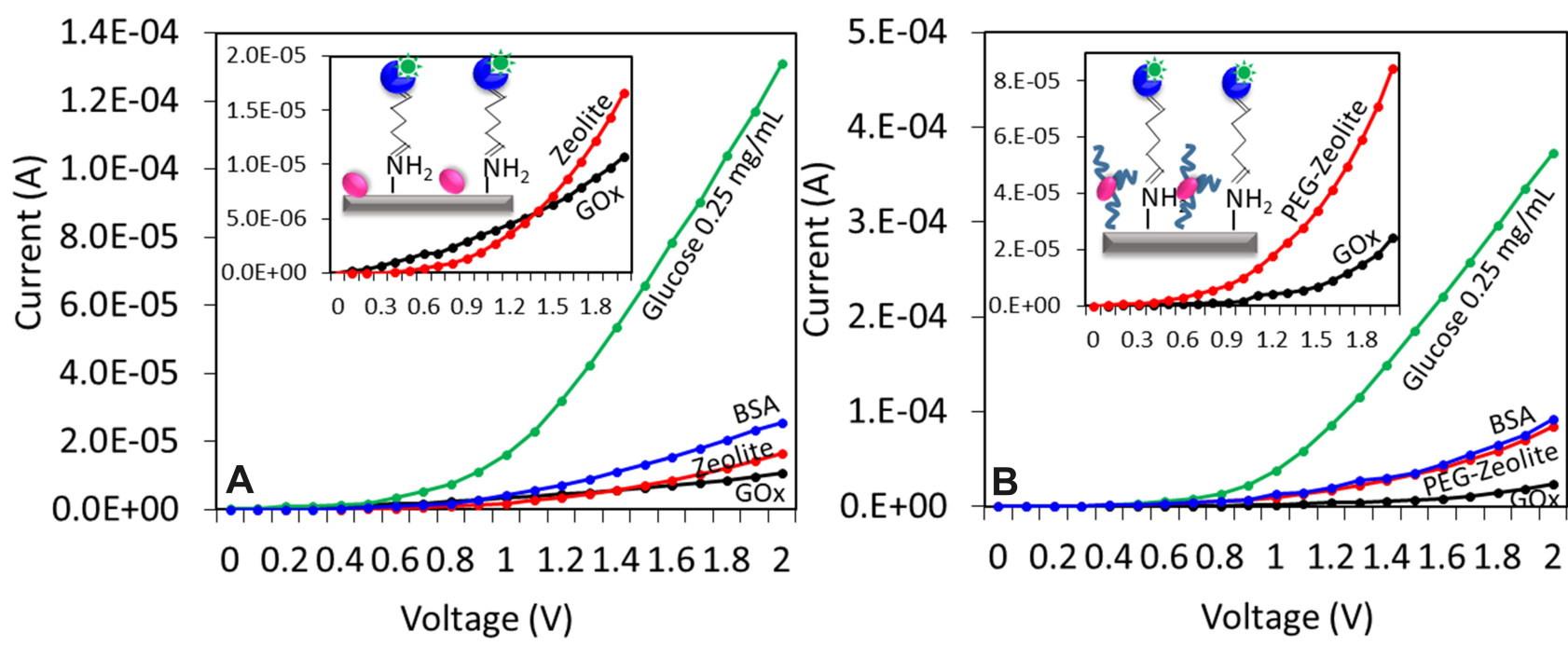

Figure 6 Comparison of glucose detection on (A) zeolite- and (B) PEG-zeolite-modified IDE surfaces. On APTES-GLU surfaces, GOx was added and bonded with GLU. The excess IDE surfaces were blocked by zeolite or PEG zeolite, and then glucose was added. The PEZ-zeolite surface shows a higher current response than that of the zeolite-modified surface with a similar concentration of glucose. Figure insets display the diagrammatic representations. 

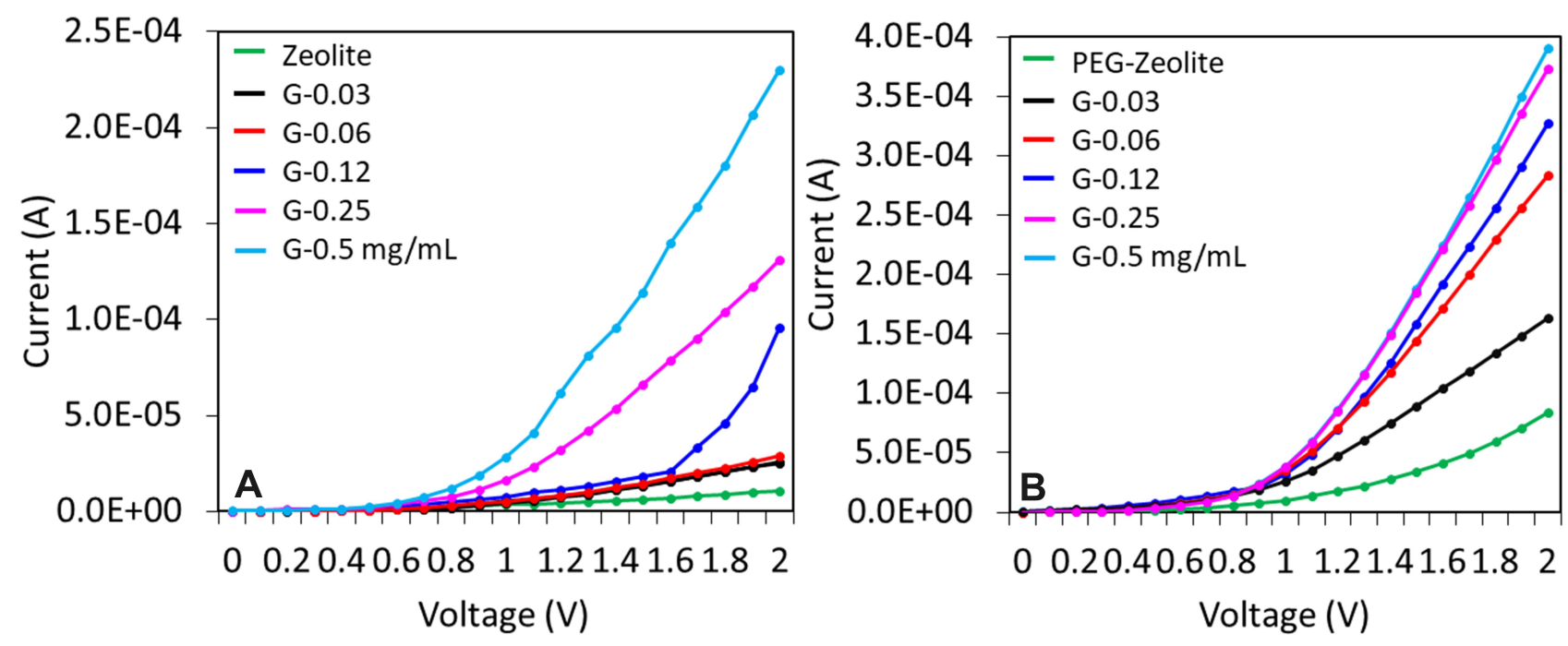

Figure 7 Comparison of the limits of glucose detection on (A) zeolite- and (B) PEG-zeolite-modified IDE surfaces. Glucose concentrations from 0.03 to $0.5 \mathrm{mg} / \mathrm{mL}$ were added independently. With PEG zeolite, the current response was increased at all glucose concentrations, and the current was gradually increased by increasing the glucose concentrations.
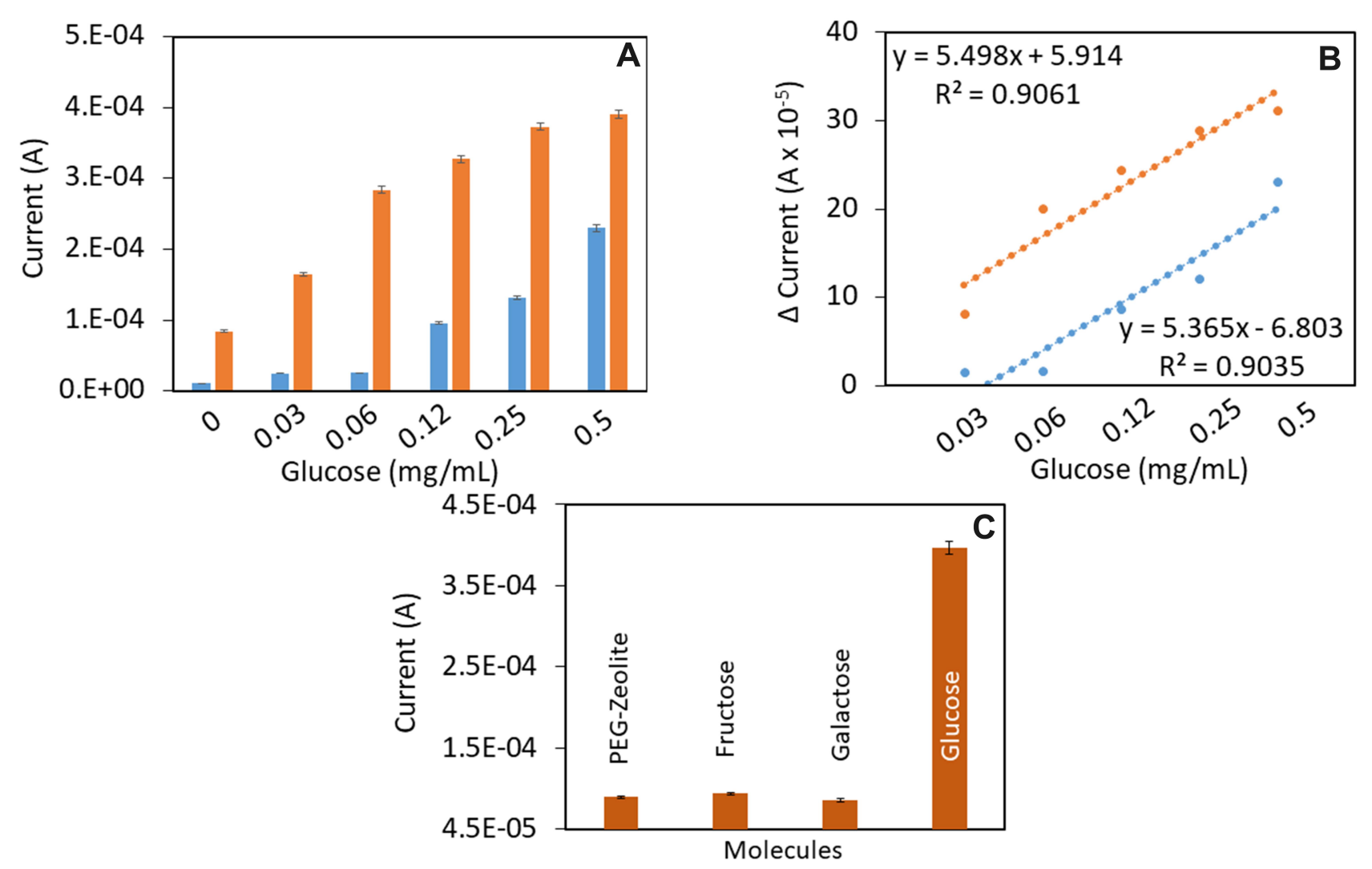

Figure 8 Comparison of current response for glucose detection on (A) zeolite and (B) PEG-zeolite modified IDE surfaces. At all glucose concentrations, the PEG-zeolitemodified surface showed a higher response in current than that of the zeolite-modified surface. (B) Linear regression analysis. Differences in the current response for glucose detection on zeolite- and PEG-zeolite-modified IDE surfaces are shown. The data were plotted in an Excel sheet, and the limit of detection was calculated. (C) Specificity of glucose detection on the PEG-zeolite modified surface. Control experiments were carried out with fructose and galactose instead of glucose. The control experiments did not show significant current changes compared with that of glucose detection. Error values were calculated by averaging the means of replicates. The p-value (0.04) was calculated by applying the null hypothesis with 6 observations. 
in current; however, from $0.12 \mathrm{mg} / \mathrm{mL}$, the current increased (Figure 7A). However, in the case of PEG zeolite, even a glucose concentration of $0.03 \mathrm{mg} / \mathrm{mL}$ showed a clear difference in the current (from $2.42 \mathrm{E}^{-5}$ to $1.64 \mathrm{E}^{-4}$ A). Moreover, the saturation of the glucose and GOx interaction showed similar current levels at 0.25 and $0.5 \mathrm{mg} / \mathrm{mL}$ glucose (Figure 7B). This result indicated that PEG zeolite not only mitigated biofouling but also improved the detection limit.

\section{High-Performance Analysis: LOD and Selectivity}

From the above results, a comparison analysis was conducted and found that PEG-zeolite-modified surface was able to perform better than the zeolite-modified surface (Figure 8A). Furthermore, the differences between the subsequent concentrations tested were subjected to linear regression analysis, and the outputs were $\mathrm{y}=5.365 \mathrm{x}$ $-6.803 ; \mathrm{R}^{2}=0.9035$ (zeolite surface) and $\mathrm{y}=5.498 \mathrm{x}+$ $5.914 \mathrm{R} 2=0.9061$ (PEG-zeolite surface) (Figure 8B). The limits of glucose detection were calculated to be $0.03 \mathrm{mg} /$ $\mathrm{mL}$ and $0.08 \mathrm{mg} / \mathrm{mL}$ for the zeolite- and PEG-zeolitemodified surfaces, respectively. The limit of detection was estimated to be the lowest concentration of the target that was detectable against the background signal $(\mathrm{S} / \mathrm{N}=$ $3: 1 ;$ LOD $=$ standard deviation of the baseline $+3 \sigma$. This showed an $\sim 3$-fold enhancement with the addition of PEF, and the sensitivities were found to be 0.03 and $0.06 \mathrm{mg}$ / $\mathrm{mL}$ for the PEG-zeolite- and zeolite-modified surfaces, respectively, displaying 2 -fold differences. The specificity for the detection of glucose was determined by testing the detection of similar sugars, namely, fructose and galactose. Neither of these sugars (at $0.5 \mathrm{mg} / \mathrm{mL}$ ) showed significant signal changes when utilizing the surface modified with PEG zeolite. By comparison, glucose displayed a clear current change and was successfully distinguished from the other sugars (Figure $8 \mathrm{C}$ ). The p-value was calculated by applying the null hypothesis with 6 observations, and the obtained p-value was 0.04 .

\section{Conclusion}

Improving biosensors with nanoparticles is attractive for diagnosing various diseases and biosensing. This work was focused on extracting zeolite nanoparticles from the coat fly ash and conducting PEGylation (PEG zeolite) to utilize the nanoparticles for sensing surface (silicon dioxide with an aluminum electrode) modification to diagnose diabetes by detecting the level of glucose. Characterization of the nanoparticles revealed the formation of zeolite with a crystalline nature and a size of $40 \pm 10 \mathrm{~nm}$ with the appropriate elemental composition. The synthesized of PEG-zeolite-modified interdigitated electrode surfaces shows great antibiofouling properties and the improvement in the detection of glucose on the dielectrode sensor surface. The designed interdigitated electrode system had a $4100 \mu \mathrm{m}$ width, $7500 \mu \mathrm{m}$ length, a $10 \mu \mathrm{m}$ gap size, 20 finger-gap pairs, a $10 \mu \mathrm{m}$ electrode size, a $4000 \mu \mathrm{m}$ finger length and a $40 \mathrm{~nm}$ electrode thickness; the electrode was operated by current-volt measurements that reflect the mechanism of the dipole moment. With this surface, the limit of detection of glucose was found to be $0.03 \mathrm{mg} / \mathrm{mL}$. Overall, the sensing surface performance was greatly improved by incorporating nanoparticles and polymers that drastically reduced the biofouling of the electrode.

\section{Disclosure}

The authors report no conflicts of interest for this work.

\section{References}

1. Kim NY, Adhikari KK, Dhakal R, Chuluunbaatar Z, Wang C, Kim ES. Rapid, sensitive, and reusable detection of glucose by a robust radiofrequency integrated passive device biosensor chip. Sci Rep. 2015;5:1-9.

2. Ge Y, Lakshmipriya T, Gopinath SCB, et al. Glucose oxidase complexed gold-graphene nanocomposite on a dielectric surface for glucose detection: a strategy for gestational diabetes mellitus. Int J Nanomedicine. 2019. doi:10.2147/IJN.S222238

3. Guo S, Li Y, Li R, et al. High-performance detection of an abdominal aortic aneurysm biomarker by immunosensing. Biotechnol Appl Biochem. 2019:1-7.

4. Lin J, Gopinath SCB, Lakshmipriya T, Chen Y, Yuan WR, Yang M. Target DNA detection of human papilloma virus-16 E7 gene by capture-target-reporter sandwich on interdigitated electrode sensor. Int $J$ Biol Macromol. 2019;141(December 2019):564-569. doi:10.1016/j.ijbiomac.2019.09.012

5. Ramanathan S, Gopinath SCB, Md Arshad MK, et al. Assorted micro-scale interdigitated aluminium electrode fabrication for insensitive electrolyte evaluation: zeolite nanoparticle-mediated micro- to nano-scaled electrodes. Appl Phys A. 2019;125(8):548. doi:10.1007/ s00339-019-2833-0

6. Ibau C, Md Arshad MK, Subash CBG, Nuzaihan M, Fathil MMF, Estrela P. Gold interdigitated triple-microelectrodes for label-free prognosticative aptasensing of prostate cancer biomarker in serum. Biosens Bioelectron. 2019;136:118-127. doi:10.1016/j. bios.2019.04.048

7. Ong CC, Siva Sangu S, Illias NM, Gopinath SC, Saheed MS. Iron nanoflorets on 3D-graphene-nickel: a 'dandelion' nanostructure for selective deoxynivalenol detection. Biosens Bioelectron. 2020;154:112088. doi:10.1016/j.bios.2020.112088

8. Letchumanan I, Gopinath SCB, Arshad MKM. Divalent ion-induced aggregation of gold nanoparticles for voltammetry immunosensing: comparison of transducer signals in an assay for the squamous cell carcinoma antigen. Microchim Acta. 2020;187:128. doi:10.1007/ s00604-020-4115-0 
9. Zhang R, Wang S, Huang X, et al. Gold-nanourchin seeded singlewalled carbon nanotube on voltammetry sensor for diagnosing neurogenerative parkinson's disease. Anal Chim Acta. 2020;1094:142-150. doi:10.1016/j.aca.2019.10.012

10. Wang Y, Guo Y, Lu J, et al. Nanodetection of head and neck cancer on titanium oxide sensing surface. Nanoscale Res Lett. 2020;15 (1):33. doi:10.1186/s11671-020-3262-X

11. Lu B, Liu L, Wang J, Chen Y, Li Z, Gopinath SCB. Detection of microRNA-335-5p on an interdigitated electrode surface for determination of the severity of abdominal aortic aneurysms. Nanoscale Res Lett. 2020. doi:10.1186/s11671-020-03331-y

12. Letchumanan I, Md Arshad MK, Gopinath SCB, Rajapaksha RDAA, Balakrishnan SR. Comparative analysis on dielectric gold and aluminium triangular junctions: impact of ionic strength and background electrolyte by pH variations. Sci Rep. 2020;10(1):6783. doi:10.1038/ s41598-020-63831-w

13. Liang T, Qu Q, Chang Y, Gopinath SCB, Liu XT. Diagnosing ovarian cancer by identifying SCC-antigen on a multiwalled carbon nanotube-modified dielectrode sensor. Biotechnol Appl Biochem. 2019. doi: $10.1002 /$ bab. 1808

14. Cheen OC, Gopinath SCB, Perumal V, et al. Aptamer-based impedimetric determination of the human blood clotting factor IX in serum using an interdigitated electrode modified with a $\mathrm{ZnO}$ nanolayer. Microchim Acta. 2017;184(1):117-125. doi:10.1007/s00604-0162001-6

15. Theodore L, Kunz RG. Nanotechnology: environmental implications and solutions. Nanotechnology. 2005;1-378.

16. Dalila RN, Md Arshad MK, Gopinath SCB, Norhaimi WMW, Fathil MFM. Current and future envision on developing biosensors aided by 2D molybdenum disulfide (MoS 2) productions. Biosens Bioelectron. 2019;248-264. doi:10.1016/j.bios.2019.03.005

17. Taniselass S, Arshad MKM, Gopinath SCB. Graphene-based electrochemical biosensors for monitoring noncommunicable disease biomarkers. Biosens Bioelectron. 2019;130:276-292. doi:10.1016/j. bios.2019.01.047

18. Ramanathan S, Gopinath SCB, Md Arshad MK, Poopalan P, Anbu P, Lakshmipriya T. Aluminosilicate nanocomposites from incinerated Chinese holy joss fly ash: a potential nanocarrier for drug cargos. Sci Rep. 2020;10(1):1-14. doi:10.1038/s41598-020-60208-x

19. Ramanathan S, Gopinath SCB, Arshad MKM, et al. Alkalinized extraction of silica-aluminium nanocomposite from traditional Chinese joss paper: optical characterizations. Mater Chem Phys. 2020;243(September 2019):122621. doi:10.1016/j. matchemphys.2020.122621

20. Vilaça N, Totovao R, Prasetyanto EA, et al. Internalization studies on zeolite nanoparticles using human cells. J Mater Chem B. 2018;6 (3):469-476. doi:10.1039/C7TB02534C
21. Rahimi M, Ng EP, Bakhtiari K, et al. Zeolite nanoparticles for selective sorption of plasma proteins. Sci Rep. 2015;5. doi:10.1038/ srep 17259

22. Knop K, Hoogenboom R, Fischer D, Schubert US. Poly(ethylene glycol) in drug delivery: pros and cons as well as potential alternatives. Angew Chem Int Ed. 2010;49(36):6288-6308. doi:10.1002/anie.200902672

23. Anniebell S, Gopinath SCB. Polymer Conjugated Gold Nanoparticles in Biomedical Applications. Curr Med Chem. 2018;1-13.

24. Hossain A. Review on PEGylation (surface coating with polyethylene glycol) and potential use of PEGylated silica-based materials for biomedical applications. J Jpn Adhes Soc. 2015;51(1):29-39.

25. Uchida K, Otsuka H, Kaneko M, Kataoka K, Nagasaki Y. A reactive poly(ethylene glycol) layer to achieve specific surface plasmon resonance sensing with a high $\mathrm{S} / \mathrm{N}$ ratio: the substantial role of a short underbrushed PEG layer in minimizing nonspecific adsorption. Anal Chem. 2005;77(4):1075-1080. doi:10.1021/ac0486140

26. Nagasaki Y, Kobayashi H, Katsuyama Y, Jomura T, Sakura T. Enhanced immunoresponse of antibody/mixed-PEG co-immobilized surface construction of high-performance immunomagnetic ELISA system. J Colloid Interface Sci. 2007;309(2):524-530. doi:10.1016/j. jcis.2006.12.079

27. Lakshmipriya T, Fujimaki M, Gopinath SCB, Awazu K, Horiguchi Y, Nagasaki Y. A high-performance waveguide-mode biosensor for detection of factor IX using PEG-based blocking agents to suppress non-specific binding and improve sensitivity. Analyst. 2013;138 (10):2863.

28. Horiguchi Y, Miyachi S, Nagasaki Y. High-performance surface acoustic wave immunosensing system on a PEG/aptamer hybridized surface. Langmuir. 2013;29(24):7369-7376. doi:10.1021/la304548m

29. Otsuka H, Nagasaki Y, Kataoka K. PEGylated nanoparticles for biological and pharmaceutical applications. Adv Drug Deliv Rev. 2012;64:246-255. doi:10.1016/j.addr.2012.09.022

30. Yao ZT, Ji XS, Sarker PK, et al. A comprehensive review on the applications of coal fly ash. Earth-Sci Rev. 2015;141:105-121. doi:10.1016/j.earscirev.2014.11.016

31. Ojha K, Pradhan NC, Samanta AN. Zeolite from fly ash: synthesis and characterization. Bull Mater Sci. 2004;27(6):555-564. doi:10.1007/BF02707285

32. Lakshmipriya T, Horiguchi Y, Nagasaki Y. Co-immobilized poly (ethylene glycol)-block-polyamines promote sensitivity and restrict biofouling on gold sensor surface for detecting factor IX in human plasma. Analyst. 2014;139(16):3977-3985. doi:10.1039/ C4AN00168K

33. Lakshmipriya T, Fujimaki M, Gopinath SCB, Awazu K, Horiguchi Y, Nagasaki Y. A high-performance waveguide-mode biosensor for detection of factor IX using PEG-based blocking agents to suppress non-specific binding and improve sensitivity. Analyst. 2013;138:2863-2870. doi:10.1039/c3an00298e
International Journal of Nanomedicine

\section{Publish your work in this journal}

The International Journal of Nanomedicine is an international, peerreviewed journal focusing on the application of nanotechnology in diagnostics, therapeutics, and drug delivery systems throughout the biomedical field. This journal is indexed on PubMed Central, MedLine, CAS, SciSearch ${ }^{\mathbb{R}}$, Current Contents ${ }^{\mathbb{B}} /$ Clinical Medicine, $^{2}$
Journal Citation Reports/Science Edition, EMBase, Scopus and the Elsevier Bibliographic databases. The manuscript management system is completely online and includes a very quick and fair peer-review system, which is all easy to use. Visit http://www.dovepress.com/ testimonials.php to read real quotes from published authors. 